\title{
The research of compression and energy absorption property of Ti6Al4V porous structure based on selective laser melting
}

\author{
Hailiang Wang*, David Zhang, Peng Zhang, Zhihao Ren \\ State Key Laboratory on Mechanical Transmission, \\ Chongqing University, \\ Chongqing 400044, China \\ 18883724859@163.com
}

Abstract-Ti6Al4V porous structure was fabricated by selective laser melting. Through the compression experiment, compression strength and energy absorption characteristics of Ti6Al4V porous structure with different rod diameters were studied. The results show that stress - strain curve of the porous structure of Ti6Al4V is divided into three stages: elastic stage, platform stage and close-grained crushing stage. The compression performance and energy absorption capacity of porous structure dramatically climbed with the increase of the rod diameter.

Keywords-selective laser melting, porous structure, compression performance, energy absorption

\section{INTRODUCTION}

As a kind of multi-functional material, porous structure which has the advantages of light weight, high specific strength, energy absorption, noise reduction, heat insulation and other excellent properties, is widely used in aviation, aerospace, automotive, mold, medical and other fields [1, 2]. Selective laser melting technology (SLM) is a new additive manufacturing technology. Unlike the traditional manufacturing, SLM is able to manufacture parts with complex internal structures, allowing us a higher degree of freedom in design, consequently leading to a cut-down in design period [3] In short, the SLM technology is an excellent approach for manufacturing porous structure.

At present, there are a lot of researches on porous structure, but few of them focus on the porous structure fabricated by selective laser melting. Chunze Yan [3] et al evaluated the manufacturability and performance of SLM produced periodic cellular lattice structures and investigated the effect of unit cell size on the manufacturability, density and compression properties of the manufactured cellular lattice structures. Chunze Yan [4] et al evaluated the manufacturability and performance of AlSi10Mg periodic cellular lattice structures with "diamond" units. Chunze Yan [5] et al investigated the manufacturability and performance of advanced and lightweight stainless steel cellular lattice structures fabricated via selective laser melting (SLM). However, few researches of compression performance and energy absorption characteristics of the Ti6Al4V diamond unit cell porous structure is could be found. Therefore, based on static compression test, this essay take a closer look at some fundamental properties of Ti6Al4V

\section{David Zhang}

College of Engineering, Mathematics and Physical Sciences, University of Exeter, North Park Road, Exeter EX4 4QF, UK

diamond unit cell porous structure by SLM, including compression modulus, compression strength, and energy absorption properties.

\section{EXPERIMENTAL}

\section{A. Materials}

Porous structure was fabricated by selective laser melting equipment-the German EOS M280. The cell type of the porous structure is the diamond cell, as shown in Fig. 1 and the material is Ti6Al4V metal powder with the particle size at 3 $50 \mu \mathrm{m}$. The size of the designed model is $\varnothing 12 \mathrm{mmx} 9 \mathrm{~mm}$; the diameter of the rod can be divided into five groups: $0.6 \mathrm{~mm}$, $0.8 \mathrm{~mm}, 1.0 \mathrm{~mm}, 1.2 \mathrm{~mm}, 1.4 \mathrm{~mm}$; the cell unit is divided into two groups of $4.5 \mathrm{~mm}$ and $5.5 \mathrm{~mm}$. A fabricated model is shown in Fig. 2.

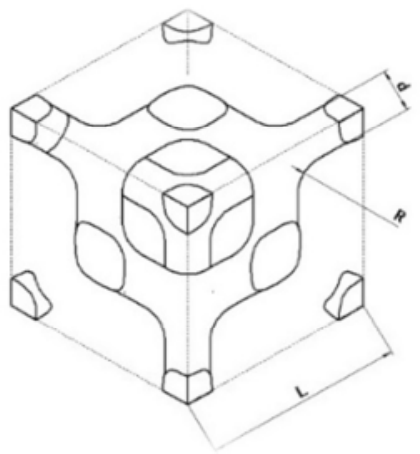

Fig. 1. Diamond cell.

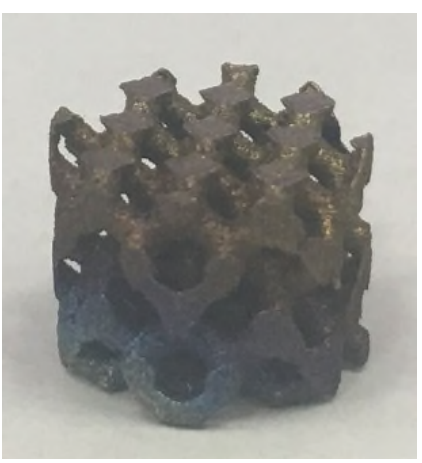

Fig. 2. The porous structure of diamond sample. 


\section{B. Experiment Method}

The compression experiment was carried out by SANS CMT5105 universal testing machine. The compression speed was at $1 \mathrm{~mm} / \mathrm{min}$ and the experimental data were recorded automatically by computer. Then the data of load and displacement are processed to obtain the data of stress and strain, and then the stress-strain curve is obtained.

The ratio of load imposed on the specimen and the cross sectional area o is referred to as apparent stress, denoted as $\sigma$. Apparent strain indicates, the ratio of deformation of specimen at length and the initial length of specimen, denoted as $\varepsilon$. Therefore, we can got $\sigma-\varepsilon$ curve, namely stress strain curve.

\section{RESULTS AND DISCUSSION}

\section{A. The Compression Stress Strain Curve of a Single Porous Structure}

From the stress - strain curve in Fig. 3, we can find that the curve can be divided into four stages.

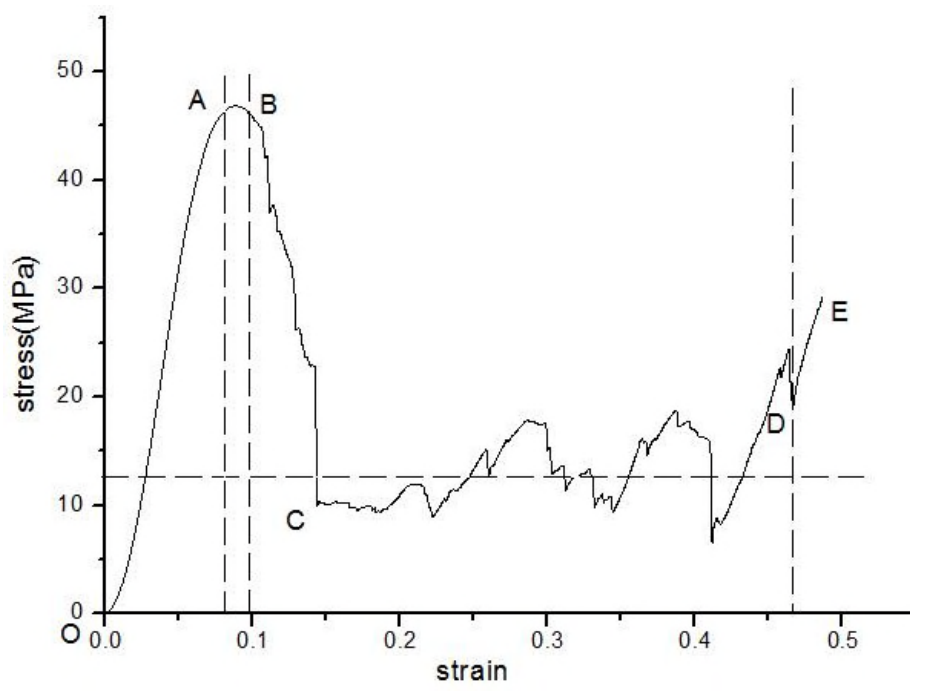

Fig. 3 Porous structure stress - strain curve of sample.

1) Elastic stage. In the process of loading, at the start of OA stages, the curve of the cellular lattice structures mainly shows an elastic region with a relative high degree of linearity.

2) Yield stage. In the $A B$ stage, following the elastic region, the specimen underwent a period of plastic deformation. The curve basically presents a straight line, and stress basically remains nearly unchanged when strain increases;

3) Fluctuating stage. In the BC stage, the porous specimen begins to break as the strain increases. The porous specimen is destroyed at one point, then the stress falls dramatically as strain increases a little. The obvious trend can be found that the stress undergoes a rapid decline with the strain increasing.

As the strain increases further, the stress still keeps changing. In the $\mathrm{CD}$ stage, the curve fluctuates fiercely with a general rule that the stress goes up as the strain increases and is followed by a sharp drop when it comes to an extreme value.
However, owing to the relatively lower broken force than that in the $\mathrm{AB}$ stage, the stress fluctuates at certain line as shown in Fig. 4.

4) Densification stage. In the end, namely the DE stage, with the strain increasing, the stress continues to increase, until the porous sample is compaction or crushing.

\section{B. The Stress - Strain Curve of the Porous Structure with Different Rod Diameters and Unit Sizes}

Figs. 4 and 5 indicate that compression strength and compression modulus of porous structure increase with the increase of rod diameter, if we set the unit cell size as an invariant. This is because the volume fraction of the porous structure becomes higher as the bar diameter increases. Consequently, the overall structure gets firmer.

By comparing the curves of Fig. 4 and Fig. 5, it can conclude that when rod diameter keeps still, compression strength and compression modulus of porous structure become higher with decrease of the size of unit cell. This is because when the unit cell size is reduced, the length of the rod between the nodes becomes shorter. So the porous structure contains more nodes, resulting in the increase of the volume fraction with the overall structure firmer.

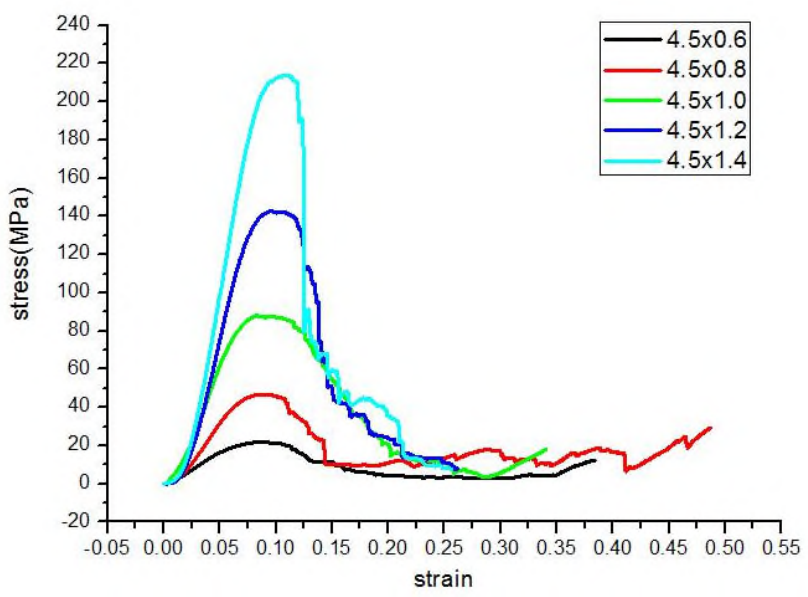

Fig. 4. The porous structure stress - strain curve of unit cell size of $4.5 \mathrm{~mm}$.

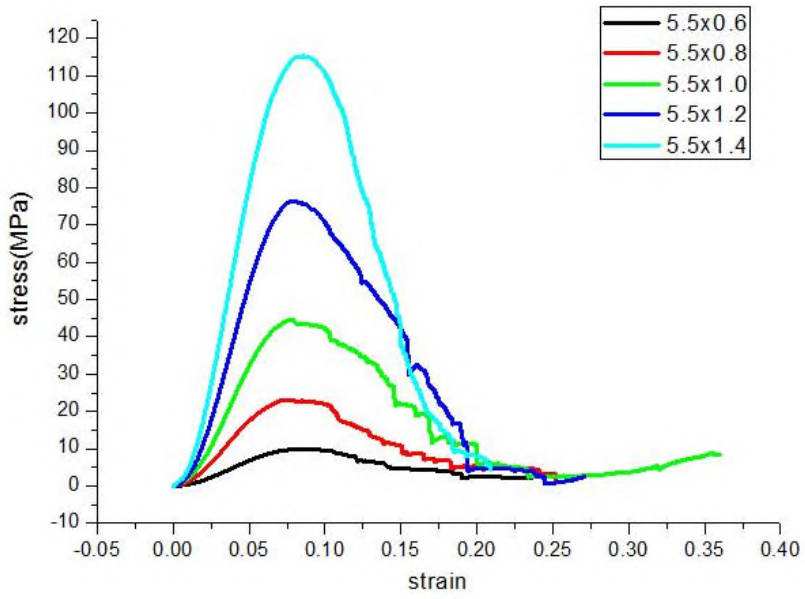

Fig. 5. The porous structure stress - strain curve of unit cell size of $5.5 \mathrm{~mm}$. 


\section{The Performance of Energy Absorption}

When the porous structure is compressed, its rod gets bent and broken with large deformation space, so it has excellent energy absorption and absorption characteristics. Energy absorption performance refers to the energy absorbed by a porous structure of a unit volume when it comes to a certain amount of deformation. The formula is as follows: [6]

$$
\mathrm{E}=\int_{0}^{\varepsilon} \sigma d \varepsilon
$$

E is the energy absorbed by the sample, and $\sigma$ is the stress of the sample, and $\varepsilon$ is the strain of the sample.

In this article, the strain is calculated from 0 to 0.2 , so the formula (1) is as follows:

$$
\mathrm{E}=\int_{0}^{0.2} \sigma d \varepsilon
$$

So the energy of static compression absorption of each experimental specimen can be calculated and then we get the curve of energy absorption of unit volume of porous structure sample. As shown in Fig. 4.

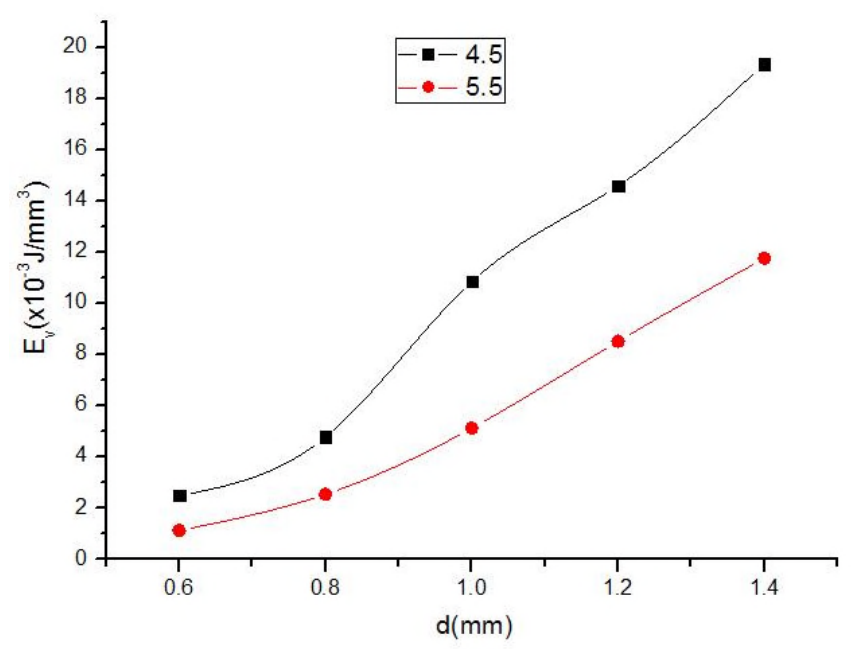

Fig. 6 The energy absorption of unit volume of porous structure sample.

According to the energy absorption curve shown in Fig. 6, it can be found when the unit cell size stays still, the energy absorption per unit volume rises with the rod diameter increases; with the same rod diameter, more energy can be absorbed by prorous structure if we increase the size of the unit cell.

\section{CONCLUSIONS}

(1) The compressive stress-strain curve of Ti6Al4V porous structure can be divided into four stages: elastic stage, yielding stage, fluctuating stage and densification stage.

(2) With the same size of unit cell, the strength and compression modulus rise as the rod diameter increase; with the same rod diameter, the smaller the unit cell, the higher strength and compressive modulus of the porous structure can be obtained.

(3) With the same unit cell, the larger rod diameter, the more energy absorbed per unit volume of the porous structure of Ti6Al4V; with the same rod diameter, the smaller size of unit cell, the more energy can be absorbed.

\section{REFERENCES}

[1] A.G. Evans, J.W. Hutchinson, N.A. Fleck, et al. "The topological design of multifunctional cellular metals". Prog. Mater. Sci. vol. 46, no. 3-4, pp. 309-327, 2001.

[2] N. Hideo, "Fabrication, properties, and applications of porous metals with directional pores". Prog. Mater. Sci. vol. 86, no. 9, pp. 884-899, 2007.

[3] C. Yan, L. Hao, A. Hussein, et al. "Evaluations of cellular lattice structures manufactured using selective laser melting". Int. J. Mach. Tool. Manuf. vol. 62, pp. 32-38, 2012.

[4] C. Yan, L. Hao, A. Hussein, et al. "Evaluation of light-weight AlSi10Mg periodic cellular lattice structures fabricated via direct metal laser sintering". J. Mater. Proc. Tech. vol. 214, no. 4, pp. 856-864, 2014.

[5] C. Yan, L. Hao, A. Hussein, et al. "Advanced lightweight 316L stainless steel cellular lattice structures fabricated via selective laser melting". Mater. Des. vol. 55, pp. 533-541, 2014.

[6] L.J. Gibson, M.F. Ashby. Cellular solids: structure and properties. City: Cambridge University Press, 1997. 